\title{
Dynamical Coulomb blockade of multiple Andreev reflections
}

\author{
A. Levy Yeyati $^{1}$, J.C. Cuevas ${ }^{1,2}$ and A. Martín-Rodero ${ }^{1}$ \\ ${ }^{1}$ Departamento de Física Teórica de la Materia Condensada CV, \\ Universidad Autónoma de Madrid, E28049 Madrid, Spain and \\ ${ }^{2}$ Institut für Theoretische Festkörperphysik, Universität Karlsruhe, D-76128 Karlsruhe, Germany
}

\begin{abstract}
We analyze the dynamical Coulomb blockade of multiple Andreev reflections (MAR) in a superconducting quantum point contact coupled to a macroscopic impedance. We find that at very low transmission the blockade scales as $n^{2}$ with $n=\operatorname{Int}(2 \Delta / e V)$, where $V$ is the bias voltage and $\Delta$ is the superconducting gap, as it would correspond to the occurrence of shots of charge ne. For higher transmission the blockade is reduced both due to Pauli principle and to elastic renormalization of the MAR probability, and for certain voltage regions it may even become an antiblockade, i.e. the current is enhanced due to the coupling with the electromagnetic environment.
\end{abstract}

PACS numbers: 73.23.-b,74.50.+r,74.45.+c,73.23.Hk

Introduction: Our understanding of coherent electron transport in superconducting nanostructures has experienced a remarkable progress in the last decade [1]. A unified picture of the dc and ac Josephson effects has emerged in which the concept of multiple Andreev reflection 2, 3] plays a central role. In this respect, superconducting atomic-size contacts have provided an ideal system in which theoretical predictions can be tested with a high degree of accuracy [4]. A remarkable feature of the MAR mechanism in superconducting quantum point contacts (SQPC) is the occurrence of coherent transfer of multiple charge quanta ne in the subgap region with $n$ increasing as $1 / V$ [5]. This is particularly striking for the case of atomic contacts where the current can then be carried by the coherent transfer of several electrons through a cross section of only few atoms.

On the other hand, the importance of charging effects in nanoscale junctions has been stressed since the early 90 's [6]. In these junctions with a very small capacitance quantum fluctuations result in a suppression of the current at low temperatures, a phenomenon known as dynamical Coulomb blockade (DCB) 7]. This effect is strongly dependent on the effective impedance of the circuit in which the nanoscale junction is embedded. One can expect these effects to scale with the charge of the carriers, which would result in a much stronger blockade when several electrons are transferred. A natural question that arises is to which extent this simple picture applies for the blockade of MAR in a SQPC. Moreover, one would like to determine the actual signatures of DCB that could be observed experimentally.

The aim of this Letter is to address these questions in the case of a single channel SQPC. For this purpose we have extended the microscopic theory of transport in the MAR regime to take into account charging effects and, more generally, the effect of quantum fluctuations in the phase introduced by the electromagnetic environment. We show that while in the tunnel limit the onset of MAR processes is strongly blocked (the relative blockade scales as $n^{2}$ ), as the transmission increases the blockade is progressively suppressed. Moreover, at high transmission one can even observe the opposite effect, i.e. an enhancement or antiblockade of the current in certain voltage regions due to the coupling with the environment.

Dynamical Coulomb blockade in a normal QPC of arbitrary transmission was analyzed in Refs [8, 9, 10]. In Ref. 8] it was shown that one can establish a direct link between DCB and shot noise in this type of systems. The relation can be written in a compact way as [1]

$$
<\delta \hat{I}>=-\frac{1}{2 e^{2}} \int d \omega J(\omega) \int d \omega^{\prime} \operatorname{sign}\left(\omega-\omega^{\prime}\right) \frac{\partial S_{I}}{\partial V}\left(\omega^{\prime}, V\right),
$$

where $<\delta \hat{I}>$ denotes the correction in the current due to DCB, $J(\omega)$ is the Fourier transform of the phase correlation function $J(t)=<\hat{\phi}(t) \hat{\phi}(0)>-<\phi^{2}>$, related to the macroscopic impedance, $Z(\omega)$, characterizing the environment by $J(t)=2 e^{2} / h \int d \omega \operatorname{Re} Z(\omega)\left(e^{i \omega t}-1\right) / \omega$ and $S_{I}(\omega, V)$ denotes the current noise spectrum of the QPC. In subsequent papers Kinderman et al. [10] extended the analysis of interaction effects to the full current distribution. In particular, in the second paper of Ref. 10] it was shown that at zero temperature and for a conductor with a linear current-voltage (IV) characteristic a relation of this type holds for the full distribution. However, as we discuss below, this relation does not hold exactly in the case of superconducting electrodes. The problem thus requires the use of more general methods.

Theoretical formalism: As discussed in Ref. [8], a QPC coupled to an environment can be described by a Hamiltonian of the form $\hat{H}=\hat{H}_{L}+\hat{H}_{R}+\hat{H}_{T}+\hat{H}_{e n v}$ where $\hat{H}_{L, R}$ correspond to the uncoupled electrodes, which we describe as BCS superconductors, $\hat{H}_{e n v}$ to the environment taken as a collection of bosonic modes [7] and $\hat{H}_{T}=\sum_{\sigma} v\left(\hat{c}_{L \sigma}^{\dagger} \hat{c}_{R \sigma} \hat{\Lambda}_{e}+\right.$ h.c. $)$ is a term coupling the leads. The translation operator $\hat{\Lambda}_{e}=e^{i \hat{\phi}}$, with $\hat{\phi}$ satisfying $[\hat{Q}, \hat{\phi}]=i e$, takes into account the change in the charge $\hat{Q}$ of the environment associated with the transfer process. Within this model the normal transmission is given by $\tau=4(v W)^{2} /\left(W^{2}+v^{2}\right)^{2}$, where 
$1 / W \pi$ is the density of states on the normal leads at the Fermi energy [3]; while the current operator is given by $\hat{I}=\frac{i e}{\hbar} \sum_{\sigma} v\left(\hat{c}_{L \sigma}^{\dagger} \hat{c}_{R \sigma} \hat{\Lambda}_{e}-\right.$ h.c. $)$.

The Keldysh formalism provides a general starting point to analyze charging effects not only on the mean current but on all the moments of the distribution. The main quantity to be determined is the cumulant generating function $S(\chi)=-\ln F(\chi)$ with 12 ]

$$
F(\chi)=<\hat{T}_{c} \exp \left\{-\frac{i}{\hbar} \int_{c} \hat{H}_{T, \chi}(t) d t\right\} \hat{S}_{c}\left(0, t_{0}\right)>
$$

where $\hat{T}_{c}$ is the chronological ordering operator on the Keldysh contour and $\hat{S}_{c}$ is the evolution operator of the system along the closed time loop. We take this contour to go from 0 to $t_{0}$ and back, $t_{0}$ being the observation time (assumed to be much larger than any other characteristic time). In Eq.(2) the system is coupled to a "counting field" $\chi(t)$, which enters as an additional phase factor $e^{i \chi(t) / 2}$ in $\hat{H}_{T}$. This field changes sign on the two branches of the Keldysh contour, i.e. $\chi\left(t_{ \pm}\right)= \pm \chi$. The mean current and all the cumulants $C_{n}$ of the distribution are obtained by repeated differentiation with respect to the $\chi$, i.e $\left.C_{n}=-(-2 i e)^{n} \frac{\partial^{n} S}{\partial \chi^{n}}\right\rfloor_{\chi=0}[13]$.

As in Refs. 8, 10 we assume that the external impedance is small $\left(z=Z /\left(h / e^{2}\right) \ll 1\right)$ and expand the generating function with respect to $\delta \hat{H}_{T}=\hat{H}_{T}-\hat{H}_{T}^{(0)}$, where $\hat{H}_{T}^{(0)}$ denotes the coupling term in the ideal voltage biased case. To first order in $Z$ we obtain

$$
\delta S(\chi)=-\frac{1}{e^{2}} \int_{c} d t_{1} \int_{c} d t_{2} J\left(t_{1}, t_{2}\right) K\left(t_{1}, t_{2}, \chi\right)
$$

where $K\left(t, t^{\prime}, \chi\right)=<\hat{T}_{c} \hat{I}_{\chi}(t) \hat{I}_{\chi}\left(t^{\prime}\right) e^{-\frac{i}{\hbar} \int_{c} \hat{H}_{T, \chi}^{(0)}(t) d t}>$. The correction to the mean current obtained by deriving $\delta S$ with respect to $\chi$ does not exactly coincide with Eq. (1). In the normal state, where the energy dependence of the transmission can in general be neglected, both expressions are equivalent. However, in the superconducting case Eq. (1) is only valid when $S_{I}(\omega, V)$ is sufficiently smooth in the scale $\omega_{0}$ of the typical energies characterizing the environment, which in general requires $\omega_{0} \ll \Delta$.

Applying Wick's theorem in Eq. (3) we obtain an expression for $\delta S$ in terms of the KeldyshNambu Green functions $G_{i, j}^{\alpha, \beta}\left(t, t^{\prime}, \chi\right)=-i<$ $\hat{T}_{c} \hat{\psi}_{i}\left(t_{\alpha}\right) \hat{\psi}_{j}^{\dagger}\left(t_{\beta}^{\prime}\right) e^{-\frac{i}{\hbar} \int_{c} \hat{H}_{T, \chi}(t) d t}>$, where $\hat{\psi}_{i}^{\dagger}=\left(\hat{c}_{i \uparrow}^{\dagger}, \hat{c}_{i \downarrow}\right)$ and $J^{\alpha, \beta}\left(t, t^{\prime}\right)=<\hat{T}_{c} \hat{\phi}\left(t_{\alpha}\right) \hat{\phi}\left(t_{\beta}^{\prime}\right)>-<\hat{\phi}^{2}>$. The indexes $i, j \equiv \pm 1$ denote the left and right electrodes (let $L \equiv 1$ and $R \equiv-1$ ). Then, Eq. (3) can be written as

$$
\begin{gathered}
\delta S(\chi)=\sum_{\alpha, \beta= \pm} \int_{0}^{t_{0}} d t_{1} \int_{0}^{t_{0}} d t_{2} J^{\alpha, \beta}\left(t_{1}, t_{2}\right) \times \\
\sum_{i, j \equiv \pm 1}(i \cdot j) \operatorname{Tr}\left[\hat{G}_{i, j}^{\alpha, \beta}\left(t_{1}, t_{2}, 0\right) \hat{\sigma}_{z} \hat{T}_{j, i}^{\beta, \alpha}\left(t_{2}, t_{1}, \chi\right) \hat{\sigma}_{z}\right]
\end{gathered}
$$

where $\hat{T}_{i, j}^{\alpha, \beta}\left(t_{1}, t_{2}, \chi\right)=\hat{v}_{i,-i}^{\alpha}\left(t_{1}\right) \hat{G}_{-i,-j}^{\alpha, \beta}\left(t_{1}, t_{2}, \chi\right) \hat{v}_{-j, j}^{\beta}\left(t_{2}\right)$, $\hat{v}_{i,-i}^{\alpha}(t)$ being a matrix in Nambu space associated with the hopping from $i$ to $-i$ :

$$
\hat{v}_{L R}^{\alpha}(t)=\alpha v\left(\begin{array}{cc}
e^{i \alpha \chi / 2} e^{i e V t / \hbar} & 0 \\
0 & -e^{-i \alpha \chi / 2} e^{-i e V t / \hbar}
\end{array}\right)
$$

with $\hat{v}_{L R}^{\alpha}(t)=\left(\hat{v}_{R L}^{\alpha}(t)\right)^{\dagger}$. By means of a double Fourier transform the Green functions can be expressed as $\hat{G}\left(\omega, \omega^{\prime}\right)=\sum_{n} \hat{G}_{0 n}(\omega) \delta\left(\omega-\omega^{\prime}+n e V\right)[3]$ and thus

$$
\begin{array}{r}
\delta S(\chi)=t_{0} \sum_{\alpha, \beta} \int d \omega \int d \omega^{\prime} J^{\alpha, \beta}\left(\omega-\omega^{\prime}\right) \times \\
\sum_{i, j= \pm 1}(i \cdot j) \sum_{n} \operatorname{Tr}\left[\hat{G}_{0 n, i j}^{\alpha, \beta}(\omega, 0) \hat{\sigma}_{z} \hat{T}_{n 0, i j}^{\beta, \alpha}\left(\omega^{\prime}, \chi\right) \hat{\sigma}_{z}\right] .
\end{array}
$$

To determine the Green functions entering in the evaluation of $\delta S(\chi)$ one has to solve the corresponding Dyson equation $\check{\mathbf{G}}=\check{\mathbf{g}}+\check{\mathbf{g}} \circ \check{\mathbf{v}} \circ \check{\mathbf{G}}$, where the $\circ$ product is a shorthand for summation over intermediate indexes and $\check{\mathbf{g}}$ are the unperturbed Keldysh Green functions of the uncoupled electrodes in equilibrium

$$
\check{g}_{i j}=\delta_{i j}\left(\begin{array}{cc}
n \hat{g}^{a}+(1-n) \hat{g}^{r} & n\left(\hat{g}^{a}-\hat{g}^{r}\right) \\
(n-1)\left(\hat{g}^{a}-\hat{g}^{r}\right) & -n \hat{g}^{r}-(1-n) \hat{g}^{a}
\end{array}\right)
$$

where $\hat{g}^{a}=\hat{g}^{r, *}=g \hat{I}+f \hat{\sigma}_{x}$, with $g=-\omega / W \sqrt{\Delta^{2}-\omega^{2}}=$ $(-\omega / \Delta) f$, are the advanced and retarded BCS Green functions, and $n(\omega)$ is the Fermi factor.

Results: In order to illustrate its effect on the IV characteristic we shall consider the simplest case of an environment characterized by a single mode of frequency $\omega_{0}$. A more general situation can be straightforwardly analyzed as a superposition of modes weighted by $\operatorname{Re} Z(\omega)$. Fig. 1 shows the overall behavior of the current blockade at zero temperature obtained by numerical evaluation of Eq.(6) for $\omega_{0}=0.2 \Delta$ and different values of the transmission. We also show for comparison the mean current $<\hat{I}>$ in the absence of environmental effects. The most prominent features are: i) in the tunnel limit the blockade appears around the threshold MAR voltages $2 \Delta / n$, ii) as the transmission increases the region where blockade is effective moves towards $V \rightarrow 0$, vanishing eventually for perfect transmission and iii) for high transmission and low bias the correction to the current $\langle\delta \hat{I}>$ can exhibit a sign change. These features are discussed in more detail below.

Let us first discuss the $\tau \rightarrow 0$ regime. Here, at a fixed bias voltage $V, \delta S$ is mainly determined by processes of order $n \sim 2 \Delta / e V$ in the transmission. These processes are illustrated by the different diagrams in Fig. 2, which correspond to $n=3$. Diagrams of type a) in Fig. 2 correspond to a real excitation of the environmental mode which gives a maximum contribution to $\delta S$ in the voltage range $2 \Delta / n \leq e V \leq\left(2 \Delta+\omega_{0}\right) / n$, i.e. between the 

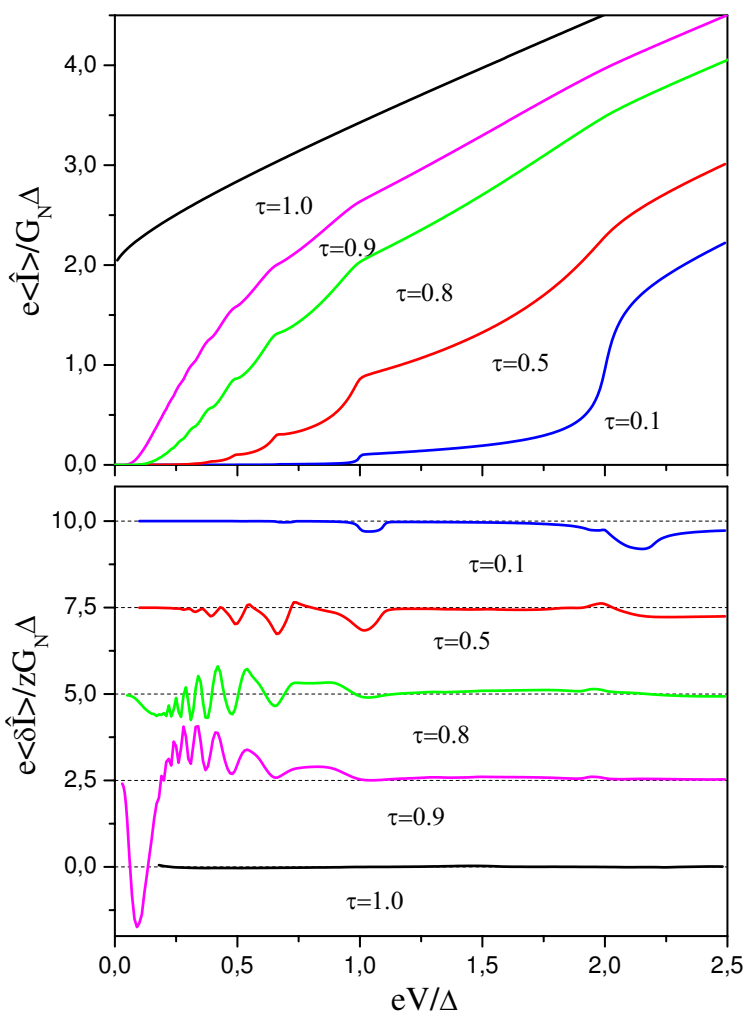

FIG. 1: (color online) Mean current (upper panel) and current blockade (lower panel) for a single mode environment with $\omega_{0}=0.2 \Delta$ at zero temperature for different transmission values. The current blockade curves have been displaced vertically for clarity. Notice normalization with $G_{N}=\left(2 e^{2} / h\right) \tau$ and $z$, which is assumed to be a small parameter.

opening of a MAR of order $n$ until the onset of inelastic processes. More precisely, their contribution to $\delta S$ at zero temperature around $e V \sim 2 \Delta / n$ is given by

$$
\begin{array}{r}
\delta S^{(a)}(\chi) \simeq-\frac{z e \pi^{2}}{h}\left(\frac{v}{4}\right)^{2 n} e^{-i n \chi} \times \\
\left\{\int_{\Delta-n e V}^{-\Delta} d \omega \rho(\omega) \rho(\omega+n e V) K_{n}(\omega, \omega)-\right. \\
\left.\int_{\Delta-n e V}^{-\Delta-\omega_{0}} d \omega \rho\left(\omega+\omega_{0}\right) \rho(\omega+n e V) K_{n}\left(\omega+\omega_{0}, \omega\right)\right\}
\end{array}
$$

with

$K_{n}\left(\omega, \omega^{\prime}\right)=\left|\sum_{j=1}^{n} \prod_{k=1}^{n-j} f(\omega+k e V) \prod_{l=n-j+1}^{n-1} f\left(\omega^{\prime}+l e V\right)\right|^{2}$,

where $\rho(\omega)=\operatorname{Im} g(\omega) / \pi$ is the BCS density of states. On the other hand, diagrams of type b) in Fig. 2 correspond to an elastic renormalization of the tunneling rates. In the tunnel limit they give a much smaller contribution which can be neglected for $\omega_{0} \ll \Delta$. From
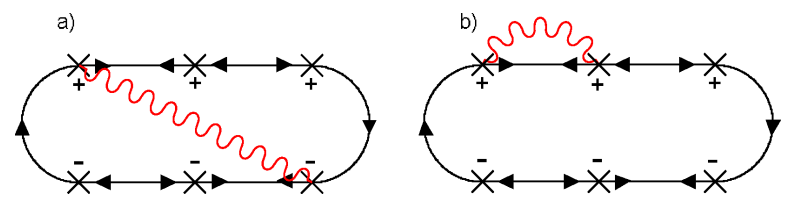

FIG. 2: (color online) Typical diagrams contributing to the blockade in the tunnel limit $e V \sim 2 \Delta / 3$. Crosses indicate the hopping events on the two branches (+ or -) of the Keldysh contour. Full lines with an arrow indicate electron or hole propagators, while double arrowed lines correspond to the anomalous ones and wavy lines denote phase correlators. Diagram a) is an inelastic correction due to the coupling with the environment while diagram b) corresponds to an elastic renormalization of the MAR probability.

Eq. (8) and taking into account that in this limit $<$ $\hat{I}>\simeq \frac{e \pi^{2}}{h}\left(\frac{v}{4}\right)^{2} n \int_{\Delta-n e V}^{-\Delta} d \omega \rho(\omega) \rho(\omega+n e V) \Gamma_{n}(\omega)$, where $\Gamma_{n}(\omega)=\prod_{k=1}^{n-1}|f(\omega+k e V)|^{2}[3]$, we find that the relative blockade $-<\delta \hat{I}>/ z<\hat{I}>$ reaches its maximum value $n^{2}$ for $2 \Delta / n \leq V \leq\left(2 \Delta+\omega_{0}\right) / n$. This result is consistent with the fact that in the tunnel limit the transmitted charge is well defined and increases in a staircase way as the voltage is reduced [5]. In this limit the environment produces a blockade of MAR processes as if they would correspond to single "shots" of charge ne until their energy is sufficiently large to excite an environmental mode.

There are, however, two basic mechanisms which reduce the blockade at finite transmissions and finite $\omega_{0}$. First, elastic renormalization of the tunneling rates (diagrams b) in Fig. 2) give a contribution to $\delta S$ which in the tunnel limit and for small frequencies can be written as

$$
\begin{gathered}
\delta S^{(b)}(\chi) \simeq \frac{z e \pi^{2}}{h}\left(\frac{v}{4}\right)^{2 n} \omega_{0}^{2}(n-1) e^{-i n \chi} \\
\left\{\int_{\Delta-n e V}^{-\Delta} d \omega \rho(\omega) \rho(\omega+n e V) \frac{\partial^{2} \Gamma_{n}(\omega)}{\partial \omega^{2}}\right\} .
\end{gathered}
$$

At the opening of the Andreev channels $\Gamma_{n}$ exhibits a minimum as a function of energy and thus the contribution to the current arising from $\delta S^{(b)}$ is positive. On the other hand, as the transmission increases there is a suppression of blockade at large bias due to Pauli principle which is closely related to the reduction observed in shot noise [5].

The evolution of the relative blockade for $\tau$ ranging from 0.01 to 0.4 is illustrated in Fig. 3. The maximum relative blockade expected in the tunnel limit is indicated by the shadowed regions. As can be observed deviations from the $n^{2}$ scaling are already significant for transmissions of the order of 0.1 .

The behavior of the blockade is qualitatively different for higher transmissions. This is illustrated in Fig. 4] which shows $\langle\delta \hat{I}\rangle$ close to the ballistic limit and for low bias voltage. It is observed that a blockade peak 


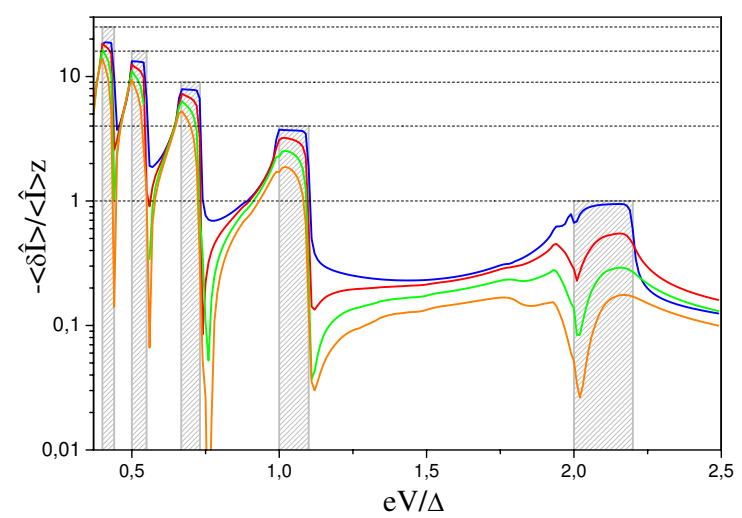

FIG. 3: (color online) Relative blockade for $\tau=0.001,0.1$, 0.2 , and 0.4 (from top to bottom) and $\omega_{0}=0.2 \Delta$. The shadowed regions indicate the maximum relative blockade (scaling as $n^{2}$ ) which is predicted for $\tau \rightarrow 0$.

at low bias is followed by a region where the system exhibits antiblockade. The overall behavior is robust with respect to changes in $\omega_{0}$ from 0 to $\sim \Delta$ (see inset in Fig. 4). One can understand this behavior from Eq.(11) which is valid when $\omega_{0} \rightarrow 0$. In this limit and at zero temperature we have $<\delta \hat{I}>\propto-\omega_{0} \frac{\partial S(0)}{\partial V}$. By virtue of this relation the blockade can be interpreted as a reaction of the system in order to compensate the power which is dissipated in the circuit by the current fluctuations introduced by the mesoscopic conductor. In a SQPC at high transmission the noise exhibits regions with negative slope $\left(\partial S_{I} / \partial V<0\right)[5]$. In these regions the compensation requires an enhancement of the current instead of a reduction. This behavior can be obtained in a simple way as follows. Close to the ballistic limit the noise at low bias arises from Landau Zener transitions between Andreev states (see second Ref. in [5]), and is given by $S(0)=\frac{2 \Delta}{V} p(1-p)$, where $p=\exp [-\pi \Delta(1-\tau) / e V]$ is the Landau-Zener probability. As a result one obtains

$$
<\delta \hat{I}>\sim \omega_{0} \frac{2 \Delta}{V^{2}} p\left[(1-p)-\frac{\pi \Delta(1-\tau)}{e V}(1-2 p)\right],
$$

which exhibits a sign change for $e V \simeq 1.75 \Delta(1-\tau)$.

Conclusions: We have analyzed dynamical Coulomb blockade in a SQPC in series with a macroscopic impedance. A scaling with the square of the effective charge is found in the limit of vanishing transmission and environmental frequencies. As the transmission increases the blockade is strongly suppressed for $e V \sim \Delta$ and for certain voltage range it may even become an antiblockade. Our predictions could be tested experimentally in superconducting atomic contacts with a well characterized electromagnetic environment, like those already used to analyze DCB in the normal state 14].

The authors would like to thank fruitful discussions with M.H. Devoret, D. Stevy and C. Urbina. JCC was

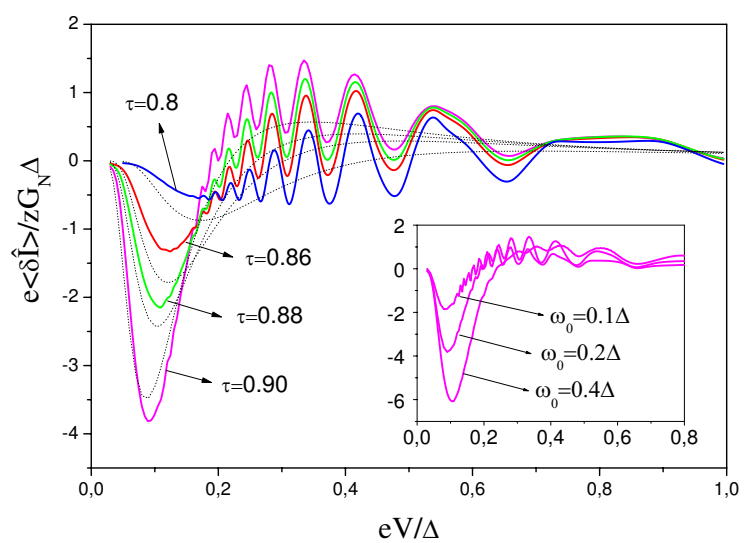

FIG. 4: (color online) Current blockade at small bias voltage and large transmission. Notice the sign change for intermediate bias. The dotted lines correspond to the approximate expression of Eq.(10). The inset shows the behavior of blockade for $\tau=0.9$ and different values of $\omega_{0}$.

financially supported by the Helmholtz Gemeinschaft.

[1] Special issue Superlattices and Microstruct. 25, No. 5/6 (1999).

[2] T.M. Klapwijk, G.E. Blonder, and M. Tinkham, Physica (Amsterdam) 109B\&110B, 1657 (1982); G.B. Arnold, J. Low Temp. Phys. 68, 1 (1987); E. N. Bratus, V.S. Shumeiko and G. Wendin, Phys. Rev. Lett. 74, 2110 (1995); D. Averin and A. Bardas, Phys. Rev. Lett. 75, 1831 (1995).

[3] J.C. Cuevas, A. Martín-Rodero and A. Levy Yeyati, Phys. Rev. B 54, 7366 (1996).

[4] E. Scheer et al., Phys. Rev. Lett. 78, 3535 (1997); E. Scheer et al., Nature 394, 154 (1998); M. Goffman et al., Phys. Rev. Lett. 85, 170 (2000); B. Ludoph et al. Phys. Rev. B 61, 8561 (2000); R. Cron et al. Phys. Rev. Lett. 86, 4104 (2001).

[5] J.C. Cuevas, A. Martín-Rodero and A. Levy Yeyati, Phys. Rev. Lett. 82, 4086 (1999); Y. Naveh and D.V. Averin, Phys. Rev. Lett. 82, 4090 (1999); J.C. Cuevas and W. Belzig, Phys. Rev. Lett. 91, 187001 (2003); G. Johansson, P. Samuelsson, and A. Ingerman Phys. Rev. Lett. 91, 187002 (2003)

[6] Single Charge Tunneling, edited by H. Grabert and M.N. Devoret (Plenum Press, New York, 1992).

[7] M.H. Devoret et al., Phys. Rev. Lett. 64, 1824 (1990); S.M. Girvin et al., Phys. Rev. Lett. 64, 3183 (1990).

[8] A. Levy Yeyati et al., Phys. Rev. Lett. 87, 046802 (2001).

[9] D.S. Golubev and A.D. Zaikin, Phys. Rev. Lett. 86, 4887 (2001).

[10] M. Kindermann and Yu. V. Nazarov Phys. Rev. Lett. 91, 136802 (2003); M. Kindermann, Yu.V. Nazarov, and C.W.J. Beenakker, Phys. Rev. B 69, 035336 (2004).

[11] M.H. Devoret (private communication).

[12] L.S. Levitov in Quantum Noise in Mesoscopic Physics, edited by Yu.V. Nazarov, Kluwer Academic Press (2002), pag. 373. 
[13] W. Belzig ibidem, pag. 463

[14] R. Cron et al. in Electron Correlations from Meso to Nano-Physics, edited by T. Martin, G. Montambaux and
J. Trân Thanh Vân, Les Ulis, France (2001), pag. 17. 